\title{
Psychisch Kranke: Warum «santésuisse» auf dem Holzweg ist
}

C. D'Souza

Santésuisse (schweizerischer Dachverband der Krankenversicherer) schreibt Schlagzeilen: ihr asozialer Vorschlag, die Krankenkassenprämie ab dem 50. Lebensjahr stetig anzuheben, weil ein Mensch ab Fünfzig rein medizinisch gesehen mehr kostet, hat zu Recht eine Welle der Empörung durchs ganze Land ausgelöst.

\section{Nur noch einmal pro Monat zur Psychiaterin?}

Doch damit nicht genug: die Santésuisse geht neue Wege, um der Kostenseite im Gesundheitswesen auf den Leib zu rücken. Sie hat im vergangenen September mittels Drohbrief unzähligen niedergelassenen FMH-Psychiaterinnen und -psychiatern in Baselland und Basel-Stadt die Wirtschaftlichkeit der Praxisführung (Wirtschaftlichkeitskontrolle) gemäss Art. 56 KVG angezweifelt und horrende Rückzahlungsforderungen angedroht für den Fall, dass der Psychiater oder die Psychiaterin nicht sofort billiger arbeitet. Im Klartext: Santésuisse legt ganz willkürlich einen Fallkostendurchschnitt von 2016.- pro Patient oder Patientin und Jahr fest, was einen «Fallbehandlungsdurchschnitt» von zwölf Sitzungen pro Jahr ergibt.

Die ganze Behandlungsfallstatistik wird von der Santésuisse nach eigenem Gutdünken besiegelt, ohne der besagten Ärzteschaft mitzuteilen, wie sie überhaupt zu dieser willkürlichen Festlegung von zwölf Therapiesitzungen pro Jahr und pro Patient oder Patientin kommt. Ausserdem wird deutlich, dass der Krankenkassenverband diese neue Form von vermeintlichen Einsparungen mit niemandem abgesprochen hat. Weder liegt ein neues KVG noch eine neue Bundesverordnung vor, welche zum Thema «Rationierung im Gesundheitswesen» besagte, dass psychisch kranke Menschen nur Dieser Artikel ist bereits in der Basler Zeitung erschienen.

Dr. med. Christine D'Souza FMH Psychiatrie \& Psychotherapie Leimenstr. 46 CH-4051 Basel
Santésuisse hat ihr Vorgehen auch nicht mit den Behörden (z.B. Sanitätsdepartement) abgesprochen, denn eine sofortige Reduktion der

\section{Anstiftung zu Kunstfehlern}

Behandlungszeit pro Patient oder Patientin würde bei einer lege artis durchgeführten Behandlung immerhin zu Unterlassungssünden und ärztlichen Kunstfehlern führen, welche gemäss $§ 4$ des Basler Medizinalpersonengesetzes schon im strafbaren Bereich zu liegen kämen. Oder anders gesagt: niemand käme auf die Idee, bei einer Hüftoperation (Totalprothese) zu verlangen, dass die Chirurginnen und Chirurgen diesen Eingriff ab sofort ohne OP-Pflegerin oder -Pfleger und bitteschön innert 20 Minuten zu erledigen hätten. Oder auch, dass eine Diabetikerin nur noch eine bestimmte Anzahl Insulineinheiten und ärztliche Diabeteskontrollen beanspruchen dürfe.

\section{Rundum Ärger}

Eine Umfrage bei anderen Psychiaterinnen und Psychiatern ergibt Folgendes: alle ärgerten sich über den Drohbrief der Santésuisse, einige nahmen bereits persönlich Stellung, einige warten die Stellungnahme der Fachgruppe «Psychiatrie» der Medizinischen Gesellschaft Basel und der Ärztegesellschaft Baselland ab und einige können nicht mehr ungestört ihrer Arbeit nachgehen, weil die anmassende Allmacht und Arroganz der Santésuisse eine schädliche Wirkung auf unsere ärztliche Arbeit ausübt. Die Tatsache, dass die Santésuisse bereits mündlich versichert hat, nichts vom Inhaltlichen (Psychiatrisch-Psychotherapeutischen) zu verstehen (!), macht die Angelegenheit noch skandalöser.

Zum Verständnis sei an dieser Stelle nachgereicht, dass die meisten Psychiaterinnen und Psychiater eine 13jährige Aus- und Weiterbildungszeit (inklusive Medizinstudium) absolviert haben und über Zusatzausbildungen verfügen, welche wiederum 3-4 Jahre in Anspruch nahmen. Ausserdem bilden sich die Psychiaterinnen und Psychiater jährlich im Durchschnitt drei bis vier Wochen weiter, was 30 bis 50\% der Ferienzeit ausmacht.

Auch sind es die Psychiaterinnen und Psychiater, die im tiefsten ärztlichen Lohnsegment arbeiten (sie verdienen weniger als ein Gymnasiallehrer mit Vollpensum) und beim Thema «Mengenausweitung im Gesundheitswesen» auf 
der Seite der kostenreduzierenden Instanzen stehen, weil sie ähnlich wie die Hausärztinnen und -ärzte über eine grosse Beziehungsfähigkeit und somit Patient(inn)entreue verfügen, was eine ärztliche Arbeit mit Wissen um Zusammenhänge ermöglicht und somit zu viel gezielteren Interventionen führt.

\section{Wirtschaftlichkeitskontrolle als Farce}

Die Santésuisse hat zugegebenermassen keine Ahnung von der Methodenvielfalt und der hohen Beanspruchung der ärztlichen Arbeit mit psychisch kranken Menschen und will nun festlegen (resp. gerichtlich erwirken), dass die Patientinnen und Patienten nur noch zwölfmal pro Jahr in die Sprechstunde kommen dürfen, rückwirkend bis ins Jahr 2001. Die Wirtschaftlichkeitskontrolle wird also zur Farce, die Santésuisse will einfach nur die Kosten im Gesundheitswesen senken und prügelt diejenige Ärztegruppe, welche die schwierigsten Patienten/-innen über lange Zeit - manchmal ein Leben lang - zu betreuen hat.

Die Patientinnen- und Patientenorganisationen sind zu dieser Ungeheuerlichkeit noch $\mathrm{zu}$ befragen und die Politikerinnen und Politiker und Behördenmitglieder müssen sich überlegen, wie viele persönlichkeitsgestörte Menschen ohne Behandlung (oder mit ungenügender Behandlung) unsere Gesellschaft erträgt. Dann werden sie sich vielleicht an die vielen «Leibachers» oder Kinderpornokonsumenten erinnern, und dann werden sich die vielen Psychosekranken, die Depressiven, die Suizidalen, die Psychotraumatisierten, die Verzweifelten, die Erschöpften, die «Ausgebrannten», die Missbrauchten, die Drogenabhängigen, die Medikamentensüchtigen und alle anderen psychisch kranken Menschen vielleicht daran erinnern, dass die Santésuisse diesen Plan in die Welt gesetzt hat, und entsprechend handeln.

In der Schweiz beenden täglich vier bis fünf Menschen ihr Leben durch Suizid. Dies sind weit mehr Tote, als es durch Unfälle oder durch AIDS zu beklagen gibt. Die Santésuisse - der Verdacht liegt nahe - scheint zu glauben, dass nur ein toter Mensch ein kostengünstiger Patient sei. Auf jeden Fall ergibt es keinen Sinn, wenn Psychiaterinnen und Psychiater bestraft werden sollen, welche psychisch kranke Menschen mit grösstem ärztlichem Know-how über manche Krise hinweg ambulant betreuen, wobei in der psychiatrischen Klinik kein Platz mehr vorhanden ist, diese Menschen stationär aufzunehmen. Die Kliniken sind - der Missstand wurde schon vor einem Jahr den Medien mitgeteilt - überfüllt, und es herrscht mehrheitlich ein Betreuungsnotstand, welcher zu noch kürzeren Klinikaufenthalten und $\mathrm{zu}$ noch anstrengenderen ambulant-psychiatrischen Behandlungen führt. Die Psychiaterinnen und Psychiater wirtschaften die Fallkostenpauschale nicht ins Bodenlose - allerdings ist unsere Gesellschaft viel kränker als man denkt, was über kurz oder lang zu entschieden höheren Kosten führen wird.

\section{Immer mehr Menschen schlucken Medikamente ohne Betreuung}

In der Schweiz verdoppelten sich in den letzten Jahren die Ausgaben für Psychopharmaka. Die betroffene Bevölkerung hingegen ist aber nicht doppelt so froh. Wie kommt das denn? Die Erklärung ist einfach: immer mehr Menschen schlucken Medikamente ohne fachgerechte Therapie und immer mehr Medikamente kosten immer mehr, d.h. die Wirtschaftlichkeit aus Sicht der Pharmaindustrie, die ist intakt, da gibt's ja auch keine Drohbriefe der Santésuisse, in denen sie beispielsweise eine Rückzahlungsforderung stellen würde wegen übersetzter Medikamentenpreise oder Schaffung von falschen Bedürfnissen.

Auch gibt es keine staatliche Instanz, die die Wirtschaftlichkeit der Santésuisse überprüft immerhin beschäftigt die Santésuisse mit ihrem Drohbrief nicht nur ihre eigenen Leute, sondern unzählige Ärztinnen und Ärzte, Anwältinnen und Anwälte, Kommissionen und Gerichte. Wer für die Folgeschäden aufzukommen hat, ist noch offen.

\section{Fische soll man nicht auf Bäumen angeln}

Immerhin, an die Adresse der Patientinnen und Patienten (jeder Mensch ist ein potenzieller Patient) seien die «zehn Gebote der Selbstverantwortung» (vgl. dazu «Beobachter» vom 12.7.02) anempfohlen, der Ärzteschaft ein beherzter, medienwirksamer Kampf gegen «krankenkässliche Dummheit» gegönnt und der Santésuisse selbst ein chinesisches Sprichwort mit auf den Weg gegeben, welches besagt, dass man Fische nicht auf Bäumen angeln soll. 